\section{Using Sources of Opportunity to Compensate for Receiver Mismatch in HF Arrays}

The spatial processing performance of adaptive sensor arrays is often limited by the nonidentical frequency responses of the receivers in the array over the passband of interest. Addressed here is the problem of estimating digital compensation for mismatches between receiver passbands in high frequency (HF) antenna arrays using interference sources of opportunity. A mathematical model of ionospherically-propagated multipath HF interference is used to develop an adaptive algorithm which estimates the receiver frequency response corrections for each receiver. The effectiveness of the proposed algorithm is experimentally demonstrated and compared against 1) a commonly used least squares technique, and 2) a highly accurate calibration system using data collected by the receiving antenna array of the Jindalee over-the-horizon (OTH) radar near Alice Springs in central Australia.

\section{INTRODUCTION}

Adaptive spatial processing is often used to mitigate cochannel interference in narrowband antenna arrays fitted with multichannel digital receivers. In practice, the cancellation performance of such techniques is often significantly limited by the nonidentical transfer functions of the receivers in the array [1-3]. For this reason, it is essential to compensate for differences between the receiver frequency responses by applying digital corrections across the passband of interest prior to adaptive spatial processing.

In the context of high frequency (HF) arrays, there is currently strong interest in using external sources of opportunity to estimate compensation for mismatches between the receiver passbands. HF sources of opportunity are normally received via the ionosphere where propagation is most often by multipath components or signal modes whose temporal and spatial characteristics are affected by the random and dynamic nature of the ionospheric reflection process. As a result of uncertain propagation effects, the precise structure of the signal mode(s) received by the antenna array are generally unknown and time varying. Standard techniques commonly

Manuscript received February 28, 1998; revised November 11, 1999 and October 24, 2000; released for publication December 4, 2000.

IEEE Log No. T-AES/37/1/02941.

Refereeing of this contribution was handled by W. D. Blair.

0018-9251/01/\$10.00 (C) 2001 IEEE used to equalize multichannel digital receivers, such as those described in [1-6] for example, rely on specific assumptions regarding the signal structure, particularly the spatial structure, and as a consequence they fail to provide suitable corrections in the multipath $\mathrm{HF}$ environment.

Section II of this article describes a mathematical model for the space-time complex-valued data aquired when the antenna array is tuned to receive a HF interference source of opportunity. An adaptive algorithm based on this model is proposed in Section III to estimate suitable compensation for receiver mismatch, this section also explains the difference between the proposed estimator and the standard least squares method. Section IV presents experimental results which illustrate the operational performance of the proposed estiamtor relative to the standard least squares method and a highly accurate radar receiver calibration system.

\section{INTERFERENCE MODEL}

A description of the experimental facility and methodology used to receive ionospherically propagated amplitude modulated (AM) radio broadcasts of opportunity with the Jindalee array can be found in [7]. The experimental data for this particular study was collected on 4 April 1998 by deliberately tuning the antenna array to a cochannel AM signal or radio frequency interference (RFI) at a carrier frequency of $13.830 \mathrm{MHz}$. This data was passively received using a frequency-modulated local oscillator which was linearly swept over a bandwidth $B=8 \mathrm{kHz}$ at a pulse repetition frequency of $f_{p}=$ $10 \mathrm{~Hz}$.

Let $\mathbf{x}_{k}(t)$ denote the complex $N$-dimensional array snapshot vector recorded at the $k$ th frequency bin (range sample) in the $t$ th pulse repetition interval (PRI). The system parameters were $N=32$ digital receivers, $K=42$ frequency bins, and $P=256$ PRI. The narrowband nature of the recorded RFI and the shortness of the PRI $\left(1 / f_{p}\right)$ compared with the typical rate of ionospheric fluctuations allow us to formulate the following general model for the output of the $n$th receiving element

$$
\mathbf{x}_{k}^{[n]}(t)=h_{k, n} \Gamma^{[n]}(t) g_{k}(t)+\mathbf{n}_{k}^{[n]}(t) .
$$

In (1), $h_{k, n}$ is the complex frequency response of the $n$th receiver at the $k$ th frequency bin, $g_{k}(t)$ is a complex scaler corresponding to the received interference waveform from a particular (reference) mode and $\mathbf{n}_{k}^{[n]}(t)$ is an additive noise component. The space-time interference modulation sequence $\Gamma^{[n]}(t)$ depends on the number of ionospheric modes $M$ and the distortions imposed upon them by the ionospheric channel. More specifically $\Gamma^{[n]}(t)$ is 
given by

$$
\begin{aligned}
\Gamma^{[n]}(t)= & \sum_{m=1}^{M} \Psi_{n}\left(f_{c}, \theta_{m}, \phi_{m}\right) \Phi_{n}\left(f_{c}\right) A_{m, n}(t) \\
& \times e^{j 2 \pi f_{c} \delta_{m}} e^{j 2 \pi\left(f_{m} / f_{p}\right) t} e^{j 2 \pi n(d / \lambda) \sin \theta_{m} \cos \phi_{m}}
\end{aligned}
$$

where $f_{m}, \theta_{m}$, and $\phi_{m}$ denote the mean Doppler shift, azimuth angle-of-arrival and elevation angle-of-arrival of the $m$ th mode, respectively, while $A_{m, n}(t)$ is a complex scaler representing the random temporal $(t)$ and spatial $(n)$ distortions imposed by the ionosphere on the $m$ th propagation mode causing Doppler spread and angular spread [8]. Due to the narrowband nature of the received interference, the waveforms arising from different propagation modes can be related to $g_{k}(t)$ by the phase shift $e^{j 2 \pi f_{c} \delta_{m}}$ where $\delta_{m}$ is the time-of-arrival of mode $m=1,2, \ldots, M$ relative to a particular (reference) mode $m_{\text {ref }}$.

The complex scalar $\Psi_{n}\left(f_{c}, \theta_{m}, \phi_{m}\right)$ represents the directional gain and phase response of the $n$th antenna sensor to the $m$ th interference mode, while the complex scalar $\Phi_{n}\left(f_{c}\right)$ represents the gain and phase response of the amplifiers and cables that connect the $n$th antenna sensor to the $n$th receiving module. The terms $\Psi_{n}\left(f_{c}, \theta_{m}, \phi_{m}\right)$ and $\Phi_{n}\left(f_{c}\right)$ vary with carrier frequency $f_{c}$ but are assumed constant over the passband of narrowband HF arrays which is typically in the order of a few kilohertz. These two terms represent the passband-independent array manifold errors or steering vector errors [9].

Although $\Gamma^{[n]}(t)$ is a random process causing some uncertainty in the signal structure, we note that this term is independent of receiver passband frequency index $(k)$. In other words, the interference snapshots recorded in each frequency bin during a particular PRI are expected to have the same (but unknown) spatial structure in the absence of frequency selective receiver mismatch $h_{k, n}$. It is this property which is exploited in the next section to estimate the receiver frequency response corrections.

\section{COMPENSATION ALGORITHM}

Let $\mathbf{c}=\left[c_{1} c_{2} \ldots c_{K}\right]^{T}$ be the complex digital corrections required to match the frequency response of an auxiliary channel " $a$ " with respect to a reference channel " $r$ ". In the frequency domain, weighting the $k$ th fast Fourier transform (FFT) output of the auxiliary channel by the $k$ th element of the correction vector $\mathbf{c}$ achieves the equalization objective. In the time domain, the inverse FFT of the elements in $\mathbf{c}$ yields the transversal filter coefficients of an equivalent equalizer based on a $K$-tap delay line.

To estimate the equalizer $\mathbf{c}$, a wideband noise source is typically injected at the reference and auxiliary receiver inputs; this mode of operation is commonly referred to as the self-cancellation mode. In this case, the least squares criterion is the mathematical foundation which is commonly used to estimate the equalizer c. Using the previously defined notation, the estimate of $\mathbf{c}$ is optimized according to this criterion by finding,

$$
\begin{aligned}
& \hat{\mathbf{c}}=\arg \min \sum_{t=1}^{P}\|\mathbf{z}(t)-\mathbf{Y}(t) \mathbf{c}\|_{F}, \\
& \hat{\mathbf{c}}=\left[\sum_{t=1}^{P} \mathbf{Y}^{H}(t) \mathbf{Y}(t)\right]^{-1} \sum_{t=1}^{P} \mathbf{Y}^{H}(t) \mathbf{z}(t)
\end{aligned}
$$

where the $K$-dimensional vector $\mathbf{z}(t)=$ $\left[\mathbf{x}_{1}^{[r]}(t) \mathbf{x}_{2}^{[r]}(t) \ldots \mathbf{x}_{K}^{[r]}(t)\right]^{T}$, the $K \times K$ diagonal matrix $\mathbf{Y}(t)=\operatorname{diag}\left[\mathbf{x}_{1}^{[a]}(t) \mathbf{x}_{2}^{[a]}(t) \ldots \mathbf{x}_{K}^{[a]}(t)\right]^{T}$ and $\|\cdot\|_{F}$ denotes the Frobenius or squared Euclidean norm. As demonstrated later, this approach is unsuitable for practical application to RFI sources of opportunity so an alternative method is now introduced. In the interests of brevity, we simply state the proposed estimator of the digital corrections as

$$
\begin{aligned}
& \tilde{\mathbf{c}}=\arg \min \sum_{t=1}^{P} \mathbf{z}^{H}(t) \mathbf{z}(t)\|\alpha(t) \mathbf{z}(t)-\mathbf{Y}(t) \mathbf{c}\|_{F} \\
& \text { subject to : } \quad \mathbf{c}^{H} \mathbf{e}=1
\end{aligned}
$$

where $\alpha(t)$ is a complex scaler and $\mathbf{e}=\left[\begin{array}{llll}1 & 0 & \ldots & 0\end{array}\right]^{T}$. This estimator differs from that in (3) due to the inclusion of a linear constraint and the two data-dependent weighting terms $\mathbf{z}^{H}(t) \mathbf{z}(t)$ and $\alpha(t)$. As shown in the Appendix, the solution of (4) can be expressed in closed form and is given by

$$
\tilde{\mathbf{c}}=\frac{\mathbf{Q}^{-1} \mathbf{e}}{\mathbf{e}^{T} \mathbf{Q}^{-1} \mathbf{e}}
$$

where $\mathbf{Q}=\sum_{t=1}^{P}\left\{\mathbf{z}^{H}(t) \mathbf{z}(t) \mathbf{Y}^{H}(t) \mathbf{Y}(t)-\mathbf{Y}^{H}(t) \mathbf{z}(t) \mathbf{z}^{H}\right.$ $\cdot(t) \mathbf{Y}(t)\}$.

\section{EXPERIMENTAL RESULTS}

1) Measurements of Receiver Mismatch: Fig. 1 shows the amplitude response of a reference and auxiliary channel measured by the Jindalee radar calibration system [8]. The ratio between the reference and auxiliary channel amplitude response is indicated by curve 3; variation of this curve over the passband is a result of frequency selective receiver mismatch. Fig. 2 shows the phase response of these two receivers (left vertical axis), while curve 3 in this figure shows the phase difference or phase mismatch between the receivers which is also passband frequency dependent (right vertical axis). The large offset of approximately $170 \mathrm{deg}$ is a result of unequal length cables connecting the Jindalee calibration source to the reference and auxiliary receivers whereas the variation of phase difference as a function of passband frequency is a result of receiver mismatch. 


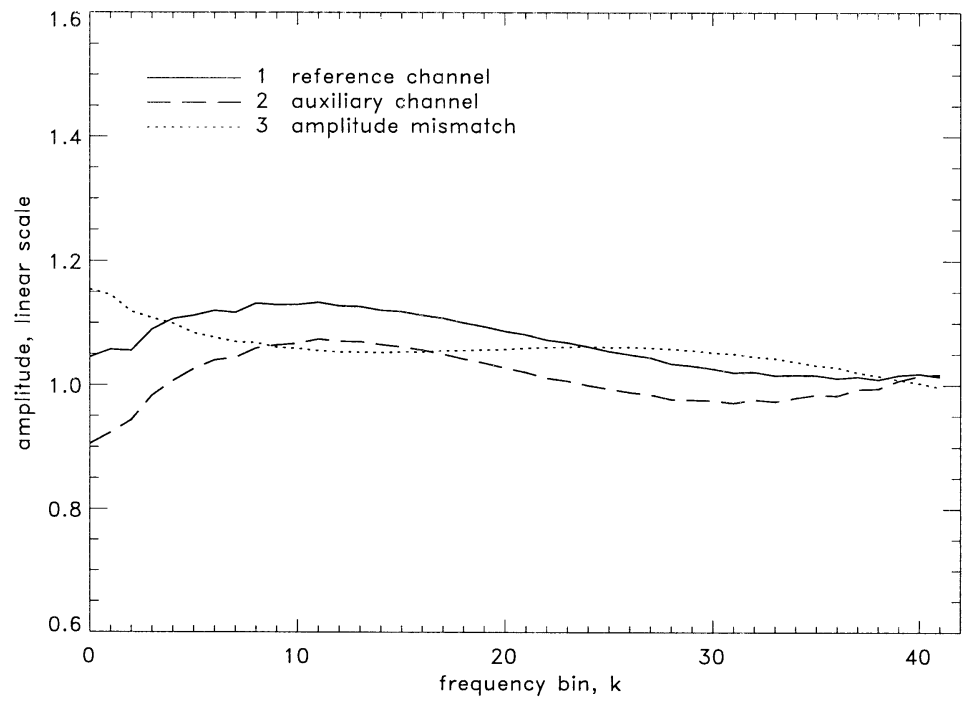

Fig. 1. Amplitude of reference channel transfer function (curve 1) and auxiliary channel transfer function (curve 2). Curve 3 shows amplitude mismatch as ratio of reference to auxiliary channel amplitude over passband of interest.

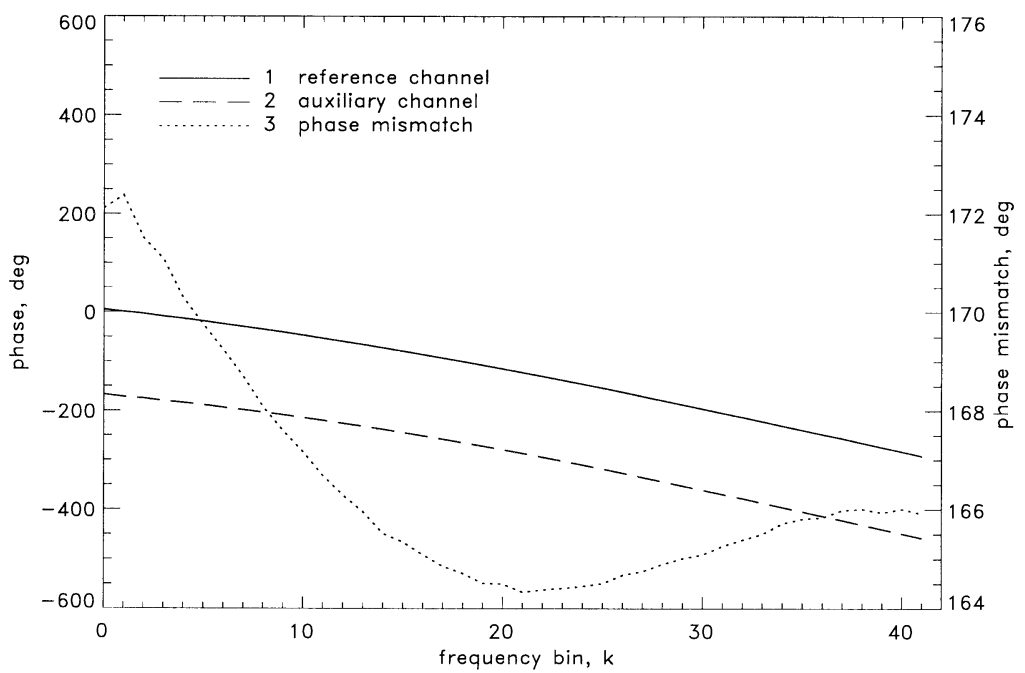

Fig. 2. Left-hand vertical axis shows phase of reference (curve 1) and auxiliary (curve 2) channel transfer functions. Curve 3 relates to right-hand vertical axis and shows difference between reference and auxiliary channel phases over passband of interest.

2) Impact of Receiver Mismatch: The deleterious effects of frequency response mismatch over all 32 receivers can be appreciated by observing the eigenvalues of the sample spatial covariance matrices $\hat{\mathbf{R}}(t)=(1 / K) \sum_{k=1}^{K} \mathbf{x}_{k}(t) \mathbf{x}_{k}^{H}(t)$ corresponding to the AM source of opportunity. As described earlier, the interference subspace of $\hat{\mathbf{R}}(t)$ is expected to have unit rank in the absence of receiver mismatch, hence the ratio between the first and second principle eigenvalues of $\mathbf{R}(t)$ is expected to be very high, especially for powerful RFI. The presence of receiver mismatch manifests itself as a reduction in this ratio, which can also be interpreted as a reduction in the spatial dynamic range of the array. To illustrate this point, Fig. 3 shows the ratio between the two principle eigenvalues of $\hat{\mathbf{R}}(t)$ as a function of pulse number $(t)$ for three different cases. Curve 1 results when the receiver transfer functions are calibrated using special signals at Jindalee. For the same data, curve 2 illustrates the significantly lower values observed for the "raw" or uncalibrated array. Curve 3 demonstrates that the eigenvalue ratio is restored after applying the frequency response corrections $\tilde{\mathbf{c}}$ estimated for all 32 receivers using the proposed method in (4).

3) Comparison of Correction Methods: Fig. 4 compares the results achieved by different estimators. Curve 1 and curve 2 are obtained by applying the compensation vectors $\tilde{\mathbf{c}}$ in (5) and $\hat{\mathbf{c}}$ in (3) to the receiver passbands, respectively (note that curve 1 is a replica of curve 3 in Fig. 3). It is clear that the proposed estimator greatly outperforms the standard (unconstrained) least squares method which hardly improves on the uncalibrated array (curve 2 in Fig. 3). 


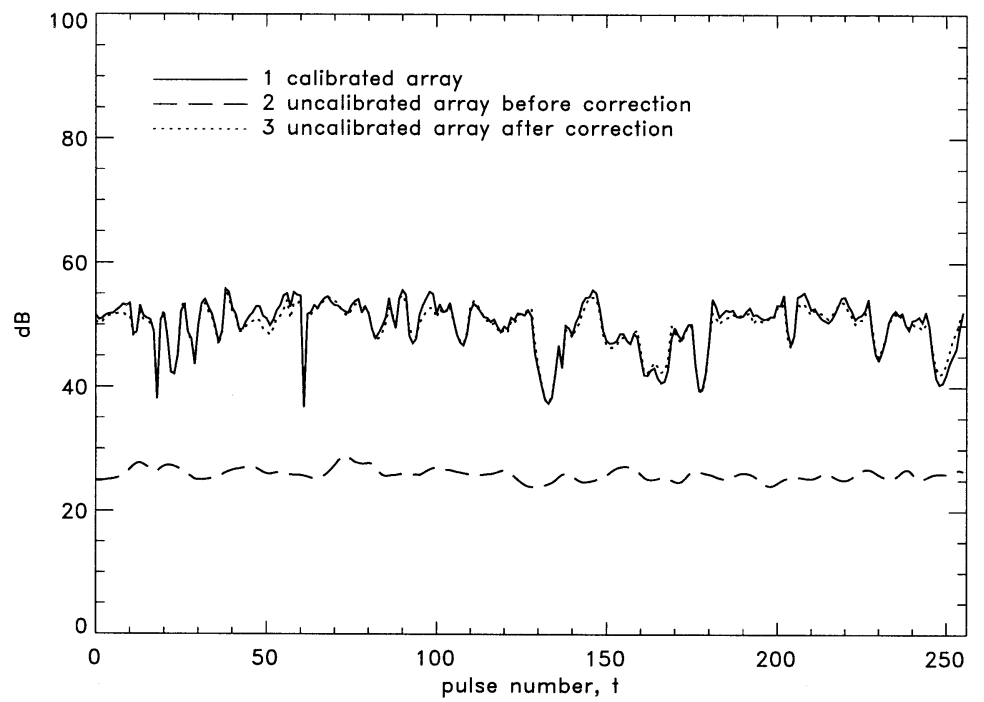

Fig. 3. Curve 1 and curve 2 show eigenvalue ratios for calibrated array and uncalibrated array, respectively. Curve 3 shows ratio when compensation estimated from source of opportunity is applied to uncalibrated array.

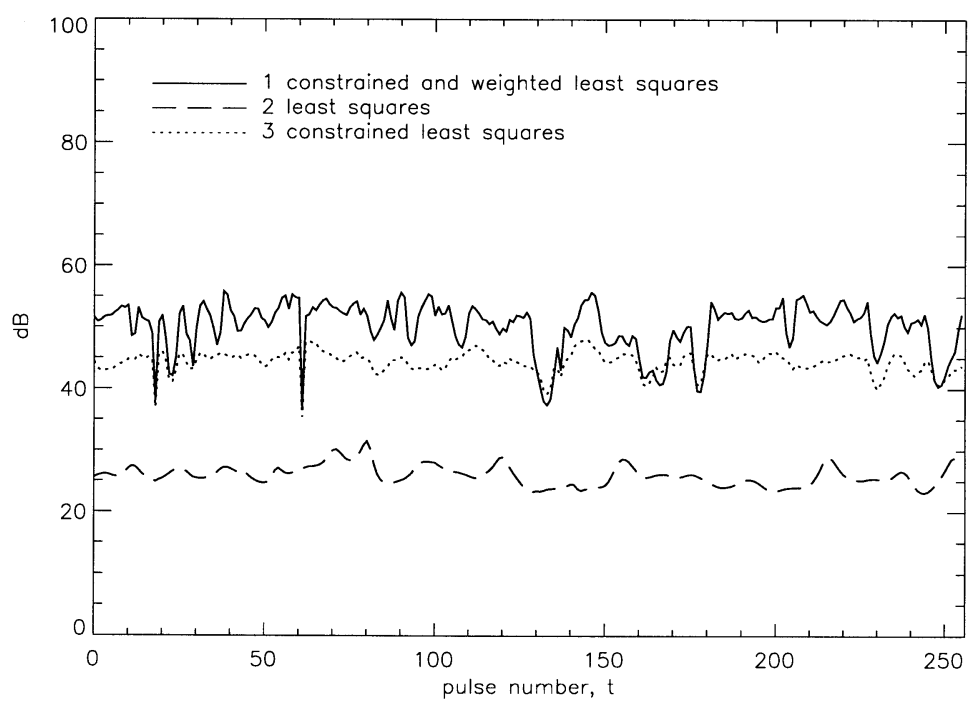

Fig. 4. Curve 1 is replica of curve 3 in Fig. 3. Curve 2 shows ratio achieved by applying compensation estimated according to unconstrained least squares criterion on (3). Curve 3 shows ratio when weighting term $\mathbf{z}^{H}(t) \mathbf{z}(t)$ is omitted from proposed estimator in (4).

Curve 3 is calculated by omitting the weighting term $\mathbf{z}^{H}(t) \mathbf{z}(t)$ in (4); the 5 to $10 \mathrm{~dB}$ loss in performance relative to curve 1 in Fig. 4 demonstrates the need for including this term to decrease estimation errors.

4) Effect of Applying Digital Corrections: Figs. 5 and 6, in the same format as Figs. 1 and 2 , demonstrate the effect of applying the digital corrections estimated by (5) to the auxiliary receiver transfer function. The flatness of curve 3 in both these figures indicates that receiver mismatch is no longer frequency selective. The frequency-independent offset between the receiver transfer functions arises from the linear constraint, but as noted in [1], such offsets are compensated by adaptive cancellation algorithms and do not effect interference rejection performance. To verify this assertion, we tuned the Jindalee array to receive a broadband interferer at a carrier frequency of $12.877 \mathrm{MHz} 12$ min prior to recording the AM source of opportunity. The spatial processing weight vector $\mathbf{w}$ for an adaptive sidelobe canceller (SLC) was estimated using the well-known sample matrix inverse technique [10] using snapshots known to contain interference and noise only.

$$
\hat{\mathbf{w}}=\frac{\hat{\mathbf{R}}^{-1} \mathbf{e}}{\mathbf{e}^{H} \hat{\mathbf{R}}^{-1} \mathbf{e}}, \quad \hat{\mathbf{R}}=\frac{2}{K P} \sum_{t=1}^{P} \sum_{k=1}^{K / 2} \mathbf{x}_{k}(t) \mathbf{x}_{k}^{H}(t) .
$$

Fig. 7 shows the Doppler spectra of the scalar SLC output $y_{k_{0}}(t)=\hat{\mathbf{w}}^{H} \mathbf{x}_{k_{0}}(t)$ for a frequency or range bin $k_{0}$ known to contain a hardware (analog) generated desired signal at a Doppler frequency of $5 \mathrm{~Hz}$. A comparison of curve 2 and curve 3 in Fig. 7 demonstrates that applying the frequency 


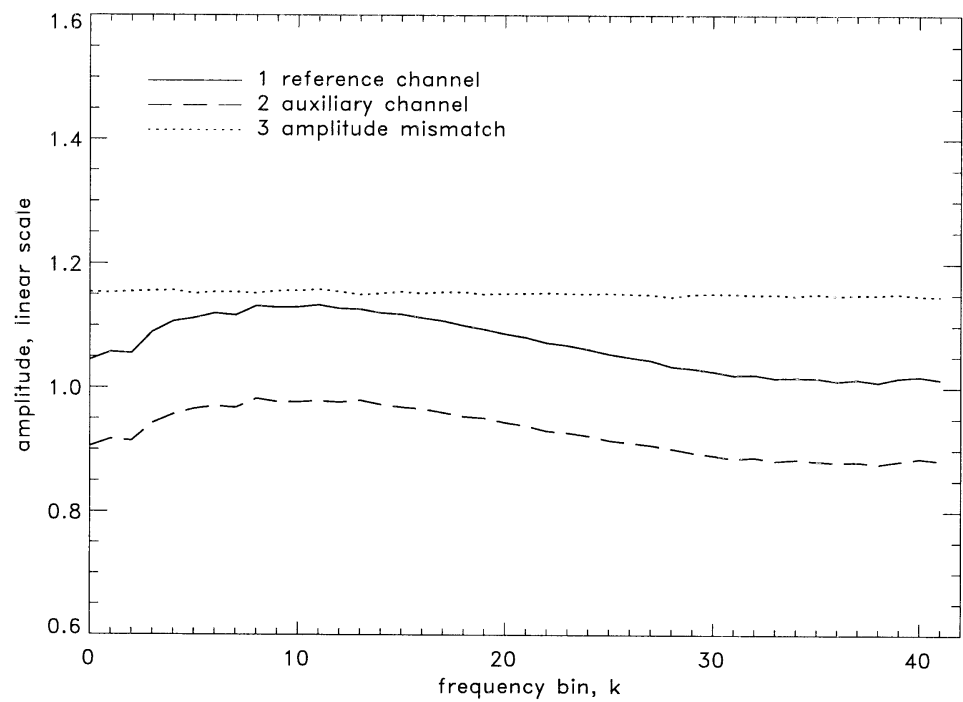

Fig. 5. Curve 1 shows amplitude of reference channel transfer function. Curve 2 shows amplitude of auxiliary channel transfer function after estimated compensation for channel mismatch is applied. Curve 3 shows amplitude mismatch between the two channels.

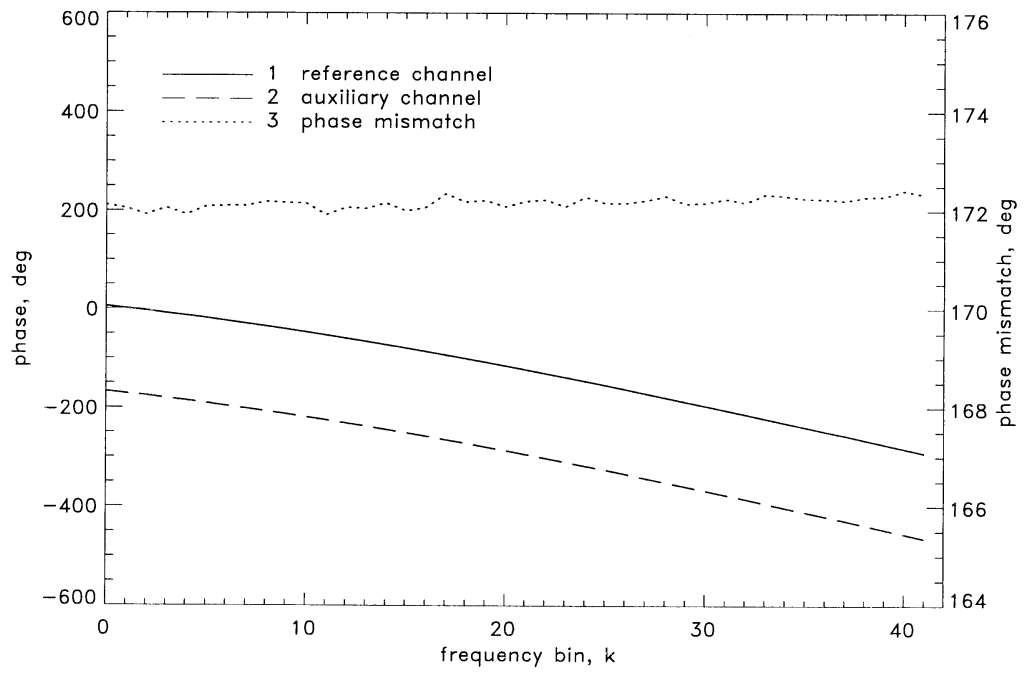

Fig. 6. Left-hand vertical axis shows phase of reference (curve 1) and compensated auxiliary (curve 2) channel transfer functions. Curve 3 relates to right-hand vertical axis and shows phase difference between the two channels after compensation is applied to auxiliary channel.

response corrections estimated by (4) improves the output signal-to-noise ratio (SNR) of the SLC by approximately $10 \mathrm{~dB}$. A comparison of curve 1 and curve 3 demonstrates that compensation for receiver mismatch estimated from RFI sources of opportunity can be used to restore the performance of an adaptive SLC to the level achieved by the highly accurate Jindalee calibration system.

\section{SUMMARY AND CONCLUSION}

This article described a space-time mathematical model for ionospherically propagated HF interference sources of opportunity and proposed an adaptive algorithm based on this model to estimate digital compensation for receiver mismatch. It was demonstrated, by direct comparison of the receiver transfer functions, that application of compensation estimated from an AM source of opportunity by the proposed method effectively removed the frequency-selective amplitude and phase mismatches inherent in 32 receivers of the Jindalee over-the-horizon $(\mathrm{OTH})$ radar array.

In addition, the proposed algorithm convincingly outperformed the commonly used (unconstrained) least squares method which, due to the presence of multipath interference components and uncertain propagation effects, was unable to remove the degradation in spatial dynamic range observed for the uncalibrated array. It was also shown that the proposed algorithm led to a $10 \mathrm{~dB}$ increase in the output SNR of an adaptive SLC operating on a broadband interference unrelated to the source of opportunity. This improvement was comparable with 


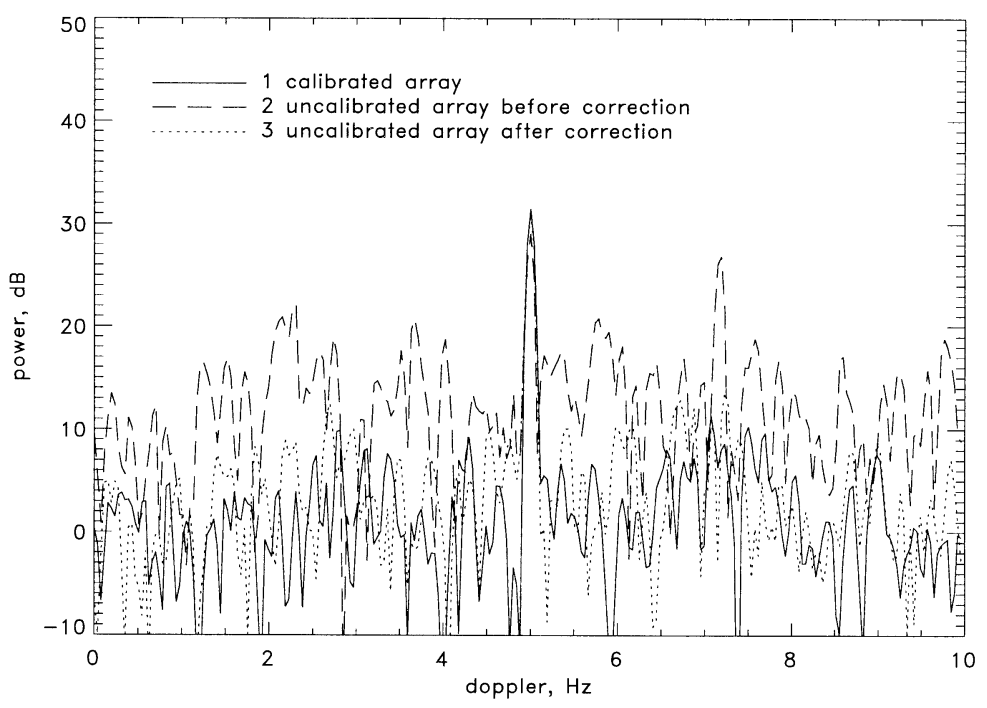

Fig. 7. Curve 1 and curve 2 show Doppler spectra corresponding to calibrated and uncalibrated array, respectively. Curve 3 shows Doppler spectrum resulting when receiver mismatch compensation estimated from source of opportunity using (4) is applied to uncalibrated array.

that achieved by sophisticated software and hardware sub-systems normally used for calibrating the Jindalee array.

Lastly, it should be noted that the multipath signal model upon which the proposed technique is based has quite general statistical properties, and for this reason, such a technique may be suitable in other adaptive sensor array applications which stand to benefit from receiver mismatch compensation prior to adaptive spatial processing.

\section{APPENDIX}

The problem is to find the argument $\tilde{\mathbf{c}}$ which minimizes the quadratic cost function $f(\mathbf{c})$ in (7) subject to the linear constraint $\mathbf{c}^{H} \mathbf{e}=1$

$$
f(\mathbf{c})=\sum_{t=1}^{P}\left\{\mathbf{z}^{H}(t) \mathbf{z}(t)[\alpha(t) \mathbf{z}(t)-\mathbf{Y}(t) \mathbf{c}]^{H}[\alpha(t) \mathbf{z}(t)-\mathbf{Y}(t) \mathbf{c}]\right\} .
$$

This problem is similar to the weighted constrained least squares optimization problems treated in [11]. However, it differs slightly from these problems because two different types of data-dependent weights are involved, namely $\mathbf{z}^{H}(t) \mathbf{z}(t)$ and $\alpha(t)$.

Let $\alpha_{\text {opt }}(t)$ be the value of $\alpha(t)$ which minimizes $\mathbf{z}^{H}(t) \mathbf{z}(t)\|\alpha(t) \mathbf{z}(t)-\mathbf{Y}(t) \mathbf{c}\|_{F}$ for an arbitrary vector $\mathbf{c}$ subject to the constraint $\mathbf{c}^{H} \mathbf{e}=1$. The value of $\alpha_{\text {opt }}(t)$ for $t=1,2, \ldots, P$ is given by

$$
\alpha_{\mathrm{opt}}=\arg \min \tilde{f}(\alpha), \quad \tilde{f}(\alpha)=(\alpha \mathbf{z}-\mathbf{Y c})^{H}(\alpha \mathbf{z}-\mathbf{Y c})
$$

where the $t$-dependence and subscripts have momentarily been dropped for notational convenience. The cost function $\tilde{f}(\alpha)$ can alternatively be written in expanded form

$$
\tilde{f}(\alpha)=\alpha \alpha^{*} \mathbf{z}^{H} \mathbf{z}-\alpha^{*} \mathbf{z}^{H} \mathbf{Y} \mathbf{c}-\alpha \mathbf{c}^{H} \mathbf{Y}^{H} \mathbf{z}+\mathbf{c}^{H} \mathbf{Y}^{H} \mathbf{Y} \mathbf{c} .
$$

To find $\alpha_{\mathrm{opt}}(t)$ we define

$$
\frac{\partial \tilde{f}(\alpha)}{\partial \alpha}=\left[\frac{\partial \tilde{f}(\alpha)}{\partial \alpha_{\mathrm{re}}}+j \frac{\partial \tilde{f}(\alpha)}{\partial \alpha_{\mathrm{im}}}\right]
$$

as the partial derivative of $\tilde{f}(\alpha)$ with respect to the complex scaler variable $\alpha=\alpha_{\text {re }}+j \alpha_{\text {im }}$. The value of $\alpha_{\text {opt }}$ is found by setting the partial derivative $\partial \tilde{f}(\alpha) / \partial \alpha$ to zero

$$
\frac{\partial \tilde{f}(\alpha)}{\partial \alpha}=\alpha \mathbf{z}^{H} \mathbf{z}-\mathbf{z}^{H} \mathbf{Y} \mathbf{c}=0 .
$$

The value of $\alpha_{\text {opt }}$ is readily derived from (10)

$$
\alpha_{\text {opt }}=\frac{\mathbf{z}^{H} \mathbf{Y} \mathbf{c}}{\mathbf{z}^{H} \mathbf{Z}} .
$$

Substituting $\alpha_{\mathrm{opt}}(t)$ for $\alpha(t)$ in (7) and simplifying the terms yields the following cost function

$$
f(\mathbf{c})=\sum_{t=1}^{P}\left\{\mathbf{z}^{H}(t) \mathbf{z}(t) \mathbf{c}^{H} \mathbf{Y}^{H}(t) \mathbf{Y}(t) \mathbf{c}-\mathbf{c}^{H} \mathbf{Y}^{H}(t) \mathbf{z}(t) \mathbf{z}^{H}(t) \mathbf{Y}(t) \mathbf{c}\right\} .
$$

The vector $\mathbf{c}$ is fixed with respect to $t$ so it can be taken out of the summation in (12). This leads to the following linearly constrained quadratic optimization problem,

$$
\tilde{\mathbf{c}}=\arg \min \mathbf{c}^{H} \mathbf{Q c} \quad \text { subject to } \quad \mathbf{c}^{H} \mathbf{e}=1
$$

where $\mathbf{Q}=\sum_{t=1}^{P}\left\{\mathbf{z}^{H}(t) \mathbf{z}(t) \mathbf{Y}^{H}(t) \mathbf{Y}(t)-\mathbf{Y}^{H}(t) \mathbf{z}(t) \mathbf{z}^{H}\right.$

$\cdot(t) \mathbf{Y}(t)\}$. This problem can be solved using the 
method of Lagrangian multipliers

$$
\tilde{\mathbf{c}}=\frac{\mathbf{Q}^{-1} \mathbf{e}}{\mathbf{e}^{T} \mathbf{Q}^{-1} \mathbf{e}}
$$

\section{ACKNOWLEDGMENTS}

The authors thank the Systems Surveillance Division at DSTO for allowing use of the Jindalee array, and acknowledge J. Ayliffe for assisting in operating the receiving system, P. Bawden and B. White for help in replaying the data, and M. Peake for useful discussions.

G. A. FABRIZIO
Surveillance Systems Division
Defence Science and Technology Organisation
P.O. Box 1500
Salisbury
South Australia 5108
Australia
E-mail: (joe.fabrizio@ dsto.defence.gov.au)
and
Dept. of Electrical and Electronic
Engineering
The University of Adelaide
South Australia, 5005
D. A. GRAY
Cooperative Research Centre for Sensor Signal
and Information Processing
SPRI Building, Warrendi Rd.
Mawson Lakes
South Australia, 5095
Australia
E-mail: (dgray@ cssip.edu.au)
and
Dept. of Electrical and Electronic
Engineering
The University of Adelaide
South Australia, 5005
M. D. TURLEY
Surveillance Systems Division
Defence Science and Technology Organisation
P.O. Box 1500
Salisbury
South Australia, 5108
Australia
E-mail: (mike.turley@ dsto.defence.gov.au)
and

\section{REFERENCES}

[1] Farina, A. (1992)

Antenna-Based Signal Processing Techniques for Radar Systems.

Norwood, MA: Artech House, 1992.

[2] Compton, R. T. (1988)

Adaptive Antennas-Concepts and Performance.

Englewood Cliffs, NJ: Prentice Hall, 1988.

[3] Monzingo, R. A., and Miller, T. W. (1980)

Introduction to Adaptive Arrays.

New York: Wiley, 1980.
[4] Johnson, J. R., Fenn, A. J., Aumann, H. M., and Willwerth, F. G. (1991)

An experimental adaptive nulling receiver utilising the sample matrix inversion algorithm with channel equlisation.

IEEE Transactions on Microwave Theory and Techniques, 39, 5 (May 1991), 798-808.

[5] Abramovich, Y. I., Kachur, V., and Struchev, V. (1984)

Methods of digital channel corrections in multichannel radar receivers.

Radio Engineering and Electronic Physics, 29, 9 (1984), 62-68.

[6] Lewis, B. L., Kretschmer, F. F., and Shelton, W. W. (1986) Aspects of Radar Signal Processing.

Norwood, MA: Artech House, 1986.

[7] Fabrizio, G. A., Abramovich, Y. I., Anderson, S. J., Gray,

D. A., and Turley, M. D. (1998)

Adaptive cancellation of nonstationary interference in HF antenna arrays.

IEE Proceedings-Radar, Sonar and Navigation, 145, 1 (Feb. 1998), 19-26.

[8] Fabrizio, G. A. (2000)

Space-time characterisation and adaptive processing of ionospherically-propagated HF signals.

Ph.D. dissertation, Adelaide University, Australia, July 2000.

[9] Compton, R. T. (1982)

The effect of random steering vector errors in the Applebaum adaptive array.

IEEE Transactions on Aerospace and Electronic Systems, AES-18, 5 (Sept. 1982), 392-400.

[10] Mallet, J. D., Reed, I. S., and Brennan, L. E. (1974)

Rapid convergence rate in adaptive arrays.

IEEE Transactions on Aerospace and Electronic Systems, 10, 6 (1974), 853-863.

[11] Albert, A. (1972)

Regression and the Moore-Penrose Pseudoinverse.

New York: Academic Press, 1972.

\section{Recognizing Occluded Objects in SAR Images}

Recognizing occluded vehicle targets in synthetic aperture radar (SAR) images is addressed. Recognition algorithms, based on local features, are presented that successfully recognize highly occluded objects in both XPATCH synthetic SAR signatures and real SAR images of actual vehicles from the MSTAR data. Extensive experimental results are presented for a basic recognition algorithm, using SAR scattering center relative locations as features with the XPATCH data and for an improved

Manuscript received October 4, 1999; revised May 8, 2000; released for publication November 17, 2000.

IEEE Log No. T-AES/37/1/02942.

Refereeing of this contribution was handled by L. M. Kaplan.

This work was supported in part by Grants F49620-97-1-0184 and F33615-99-C-1440. The contents and information do not reflect the policies and positions of the U.S. Government.

$\overline{0018-9251 / 01 /} \$ 10.00$ (C) 2001 IEEE 